\title{
HYPERCHAOS AND ITS CONTROL IN TWO-LEVEL QUANTUM OSCILLATORS LATTICE
}

\author{
Andrey Andreev \\ Neuroscience and Cognivite Technology Laboratory \\ Center for Technologies in Robotics and Mechatronics Components \\ Innopolis University \\ Russia \\ a.andreev@innopolis.ru
}

Article history:

Received 15.10.2019, Accepted 26.11.2019

\begin{abstract}
The behavior of the square lattices of coupled twolevel quantum oscillators is investigated. As quantum oscillators, we use the model of Rydberg atoms obtained using the approximation of a fully factorized density matrix. To investigate the behavior of the system the Lyapunov exponents spectra are calculated. Chaos and hyperchaos in the systems are revealed. It was shown that the number of positive Lyapunov exponents almost linearly depends on the number of atoms in the system, at a rate suggesting that adding three atoms leads to the appearance of an additional positive Lyapunov exponent. Using an external parametric effect and continuous feedback is suggested to control the complicated dynamics in the system. Using continuous feedback allows reducing the number of positive Lyapunov exponents from 3 to only 2 while introducing external parametric influence into the system allows reducing their number down to 0 and completely suppresses hyperchaos.
\end{abstract}

\section{Key words}

Rydberg atoms, chaos, hyperchaos, control, Lyapunov exponents.

\section{Introduction}

Nowadays, the problems of controlling quantum systems with Rydberg atoms are of considerable interest due to the fact that such problems are closely related to the problem of creating quantum computers [Jaksch et al., 2000; Zagoskin, 2011]. It is known that Rydberg atom is an excited atom with one or more electrons that have a very high principal quantum number around 1000 [Weimer et al., 2008; Heidemann et al., 2007]. At present, such objects are of great interest [Greenland et al., 2010; Gaëtan et al., 2009; Andreev et al., 2015;
Labuhn et al., 2016; Barredo et al., 2015] since they can be used for the quantum control of one atom by another, due to the Rydberg (highly excited) state. The size of atoms in the basic state is no higher than $0.1 \mathrm{~nm}$, while in the Rydberg state they can be as high as several nanometers or more. This allows atoms that are far enough apart to prevent their interaction while they are in the ground state to strongly interact when excited [Schwarzschild, 2009].

The problem of chaotic behavior in a quantum system is of special interest [Ivanchenko et al., 2014; Ostrovskaya and Nori, 2016; Eastman et al., 2017]. It is also interesting from a practical point of view when solving problems with quantum calculations for clusters of atoms introduced into a solid while in the Rydberg state [Saffman et al., 2010].

Such systems with Rydberg atoms are promising for storing and transmitting information. The problem of analyzing how to suppress chaotic behavior in such systems is important since chaos can damage stored or transmitted information.

In this work, we investigate the behavior of the square lattices of $N$ coupled quantum oscillators using a calculation of Lyapunov exponents spectra. As quantum oscillators, we use the model of Rydberg atoms obtained using the approximation of a fully factorized density matrix and mean-field theory. We reveal chaos in the system of $N>4$ and hyperchaos characterized by the number of positive Lyapunov exponents $M>2$ in the systems of $N>9$. We show that the number of positive Lyapunov exponents almost linearly depends on the number of atoms in the system, at a rate suggesting that adding three atoms leads to the appearance of an additional positive Lyapunov exponent. We suggest using an external parametric effect and continuous feedback to control the complicated dynamics in the system. Using continuous 


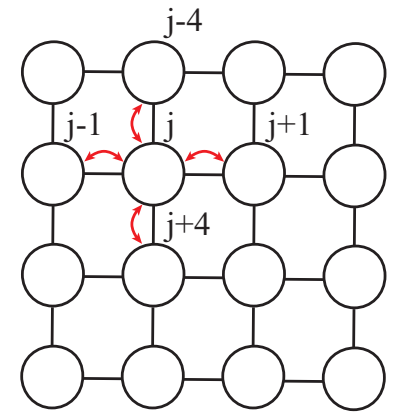

Figure 1. Schematic representation of a square lattice of 16 interacting Rydberg atoms, labelled by integer indexes and coupled via nearest neighbor interaction.

feedback allows reducing the number of positive Lyapunov exponents from 3 to only 2 while introducing external parametric influence into the system allows reducing their number down to 0 and completely suppresses hyperchaos.

\section{Mathematical Model}

A lattice of coupled Rydberg atoms is described by the following differential equations obtained in [Lee et al., 2011] using the mean-field theory and assuming that each atom has the ground and Rydberg states only [Andreev et al., 2019]:

$$
\begin{aligned}
& \dot{w}_{j}=-2 \Omega \Im q_{j}-\left(w_{j}+1\right) \\
& \dot{q}_{j}=i\left[\Delta-c \sum_{k \neq j}\left(w_{k}+1\right)\right] q_{j}-\frac{1}{2} q_{j}+i \frac{\Omega}{2} w_{j} .
\end{aligned}
$$

where $\Delta$ is the detuning between the laser and transition frequencies, $\Omega$ is the Rabi frequency (tuned by the laser field amplitude), $c$ is the Rydberg interaction, $w_{j}$ corresponds to the population inversion of the $j$-th atom, $q_{j}$ corresponds to its coherence.

In this work, we study square lattices consisting of 4 , 9, 16, 25, and 36 interacting Rydberg atoms. Schematic representation of a square lattice of 16 interacting Rydberg atoms is shown in Fig. 1. In our modeling, we suggest that each atom is connected with its neighbors only in horizontal or vertical lines, but not with diagonal elements.

\section{Hyperchaotic Behaviour}

In order to analyze the dynamics of the system, we use the calculation of Lyapunov exponents [Kuznetsov, 2001; Maximenko et al., 2017]. To calculate the spectrum of Lyapunov exponents, we introduce $3 * N$ disturbing vectors (since there are $3 * N$ variables in our system), each of which has six components and we analyze their evolution along the considered phase trajectory. In equal time periods, the vectors are renormalized and are orthogonalized by the Gramm-Schmidt procedure. As a result, we remove the effects of all previous vectors from each vector in numerical order; this allows us to calculate not just the highest exponent, but their whole spectrum. After each orthogonalization and before renormalizing, we calculate the natural logarithm for each vector and calculate the sums of logarithms for each disturbing vector. Dividing these sums over time, we obtain the Lyapunov exponents.

Figure 2 illustrates the 9 highest Lyapunov exponents for the lattice of $N=16$ Rydberg atoms for $\Omega=2.0$. One can see that for small values of $\Delta$ the stable equilibrium state exists characterized by all Lyapunov exponents are negative $\left(\Lambda_{1, \ldots, 9}<0\right)$. With increasing of $\Delta$, it switches to a periodic solution as a result of an Andronov-Hopf bifurcation at $\Delta \approx 2.0$, where $\Lambda_{1}$ becomes zero. At $\Delta \approx 2.45$ the solution undergoes a period-doubling bifurcation when $\Lambda_{2}$ approaches zero and then it follows by a cascade of period-doubling bifurcations. At $\Delta \approx 2.65$ the periodic oscillations lose their stability, resulting in the onset of hyperchaos characterized by the two highest Lyapunov exponents are positive $\left(\Lambda_{1,2}>0\right)$, the third is zero $\left(\Lambda_{3}=0\right)$ and the others are negative $\left(\Lambda_{4, \ldots, 9}<0\right)$. Then, $\Lambda_{3}$ quickly becomes positive and $\Lambda_{4}$ becomes zero making hyperchaos more complicated. Further increasing of $\Delta$ leads to increasing of positive Lyapunov exponents number up to 5. The hyperchaotic behavior persists up to $\Delta \approx 8.22$, after which it rapidly switches to the periodic solution. For $\Delta>8.45$, all oscillations disappear, and all longterm solutions in the system correspond to a stable equilibrium. In the region of hyperchaos $(2.65<\Delta<8.22)$ there are 3 windows of periodicity where the hyperchaos is replaced by complex periodic solutions. In Fig. 2 these windows are reflected by $\Lambda_{1}$ falling back to zero. The emergence of these windows of periodicity is typical for chaos and hyperchaos born through a cascade of period-doubling bifurcations.

We have analyzed lattices with $4<N<36$ and revealed that hyperchaos exists in the systems with $N>9$. It becomes more complicated as more Lyapunov exponents become positive. The number of positive Lyapunov exponents as a function of the number of atoms in the system is shown in Fig. 3. For a system of 4 interacting Rydberg atoms, there is only one positive Lya-

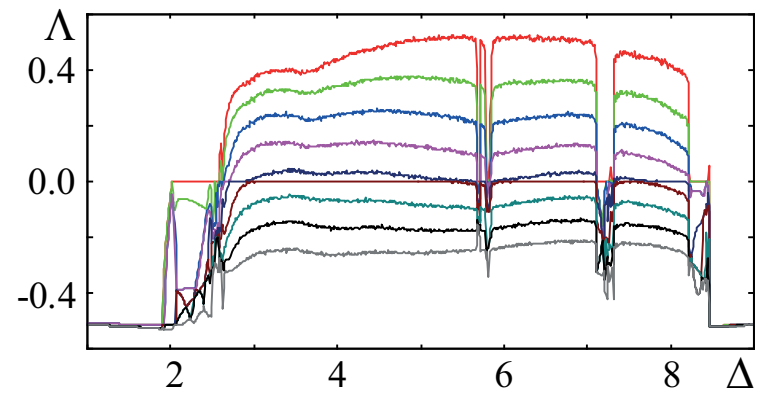

Figure 2. Lyapunov exponents spectrum for the lattice of $N=16$ Rydberg atoms for $\Omega=2, c=5$. 


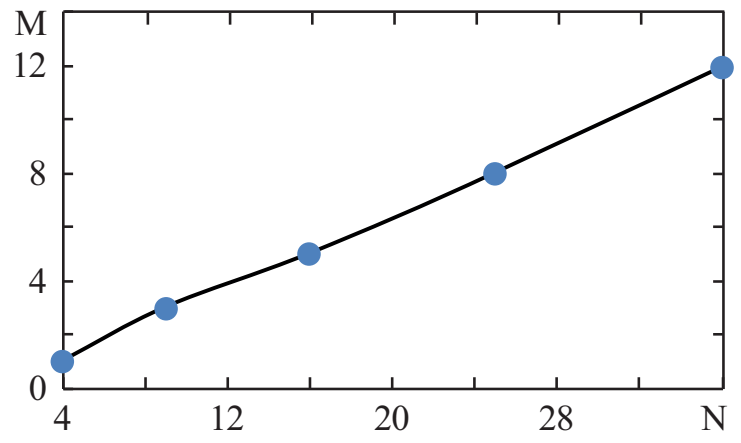

Figure 3. Number of positive Lyapunov exponents $M$ versus number of atoms $N$ in the lattice calculated for $\Delta=7, \Omega=2, c=5$.

punov exponent which means that there is only chaos exists and no hyperchaos. The graph shows an almost linear growth, at a rate suggesting that adding three atoms leads to the appearance of an additional positive Lyapunov exponent.

\section{Control}

In order to control the complicated dynamics in the system, we suggest using an external parametric effect [Mirus and Sprott, 1999] and continuous feedback [Hramov et al., 2006; Pyragas, 1995]. As the external parametric effect, we use the modulation of Rabi frequency $\Omega$ since it is possible to implement such modulation in the experiment by modulating the intensity of the laser field exciting the atoms. In the system of equations (1) that describes the system of interacting Rydberg atoms, the above external effect is written by modifying the Rabi frequency as follows

$$
\Omega=\Omega_{m}(1+M \sin (2 \pi f t))
$$

where $\Omega_{m}$ is the Rabi frequency in the autonomous system, $M$ is the depth, and $f$ is the parameter's modulation frequency.

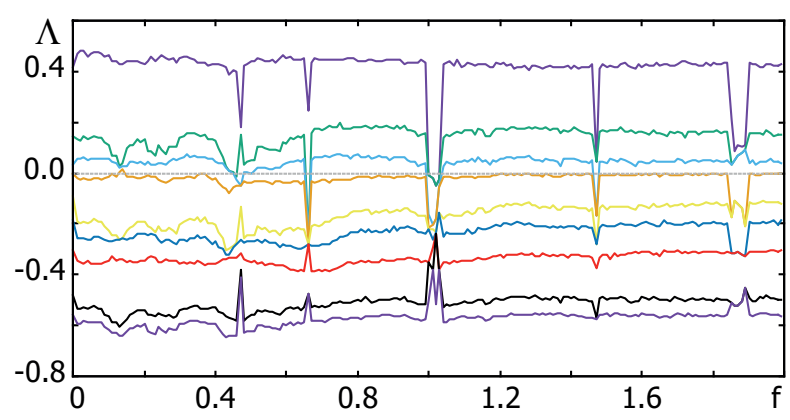

Figure 4. Variation of the nine largest Lyapunov exponents with the external effect frequency $f$ for the lattice of $N=9$ Rydberg atoms for $\Omega=2.0, \Delta=5.0$ corresponding to hyperchaos characterized by 3 positive Lyapunov exponents, $M=0.2$.

We analyze dynamics of the lattice of $N=9$ Ryd- berg atoms (1) with external parametric effect (2) for $\Omega=2.0, \Delta=5.0$ corresponding to hyperchaos characterized by 3 positive Lyapunov exponents in the autonomous system. As there are 2 parameters of external effect, firstly, we fix the modulation depth $M=0.2$ and change the modulation frequency $f$ from 0 to 2 trying to find an optimal value of $f$. To analyze the system dynamics we calculate the spectrum of conditional Lyapunov exponents [Pyragas, 1997; Hramov and Koronovskii, 2005], in which one zero exponent is missing, unlike the common Lyapunov exponents' spectrum.

Fig. 4 illustrates the variation of the nine largest Lyapunov exponents with the external effect frequency $f$ for the lattice of $N=9$ Rydberg atoms for $\Omega=2.0$, $\Delta=5.0$ corresponding to hyperchaos characterized by 3 positive Lyapunov exponents for fixed $M=0.2$. One can see the areas of $f$ where the number of positive Lyapunov exponents is decreased by $1(f \approx 0.47$, $f \approx 1.87), 2(f \approx 0.67, f \approx 1.47)$ or $3(f \approx 1.02)$ that corresponds to complete suppressing of hyperchaos in the system.

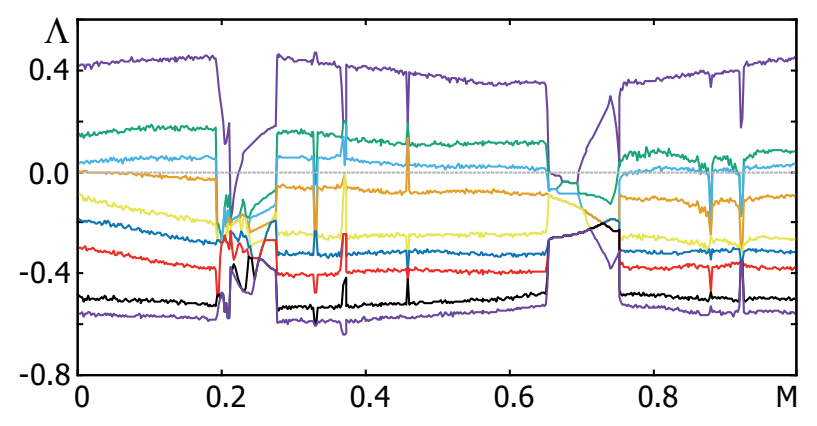

Figure 5. Variation of the nine largest Lyapunov exponents with the parameter's modulation amplitude $M$ for the lattice of $N=9 \mathrm{Ry}$ dberg atoms for $\Omega=2.0, \Delta=5.0$ corresponding to hyperchaos characterized by 3 positive Lyapunov exponents, $f=1.02$.

Then, we fix $f=1.02$ corresponding to complete suppressing of hyperchaos, characterized by 3 positive Lyapunov exponents in the autonomous system and change the modulation depth from 0 to 1 for $\Omega=2.0, \Delta=5.0$, $M=0.2$. Fig. 5 illustrates the variation of the nine largest Lyapunov exponents with the parameter's modulation amplitude $M$. One can see two ranges of values of the amplitude of the external effect $(M \approx 0.21$ and $M \approx 0.267$ ) when it is possible to achieve complete suppression of hyperchaos in the system to establish periodic dynamics in it.

The method of controlling oscillations by introducing continuous feedback is based on modulation of the intensity of the external exciting laser radiation by a signal taken from one of the atoms of this system [Pyragas, 1992]:

$$
\Omega=\Omega_{m}\left[1+M\left(w_{j}-\bar{w}_{j}\right)\right]
$$


where $\Omega_{m}$ is the Rabi frequency in the autonomous system, $M$ is an amplitude of feedback, $j$ is a number of atom which is used for feedback, $\bar{w}_{j}$ is the value of $w_{j}$ averaged for the last 5 seconds.

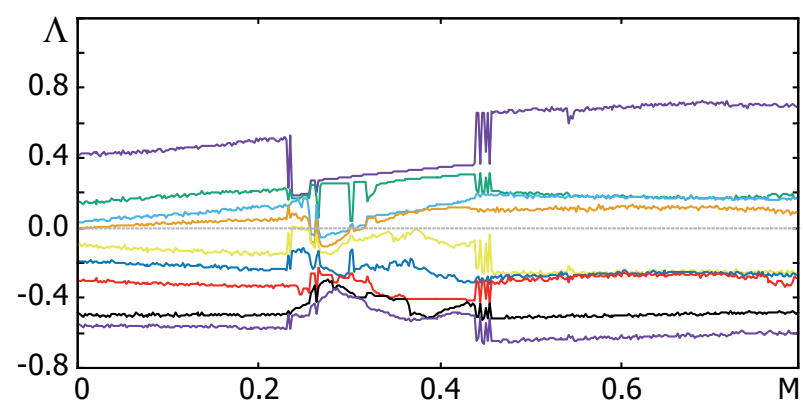

Figure 6. Variation of the nine largest Lyapunov exponents with the feedback amplitude $M$ for the lattice of $N=9$ Rydberg atoms for $\Omega=2.0, \Delta=5.0$ corresponding to hyperchaos characterized by 3 positive Lyapunov exponents.

We analyze dynamics of the lattice of $N=9$ Rydberg atoms (1) with continuous feedback (3) for $\Omega=2.0$, $\Delta=5.0$ corresponding to hyperchaos characterized by 3 positive Lyapunov exponents in autonomous system as we did for the system with external parametric effect (2). Fig. 6 illustrates variation of the nine largest Lyapunov exponents with the feedback amplitude $M$. One can see that when choosing the value of the feedback amplitude, it is possible to reduce the number of positive Lyapunov exponents from 3 to only 2 while introducing external parametric influence into the system allows reducing their number down to 0 . The latter testifies to the greater efficiency of using the method of introducing external parametric effect into the system for controlling hyperchaotic dynamics in comparison with the method of introducing continuous feedback. This is due to the fact that when using continuous feedback, a chaotic signal is supplied to the system, which makes it possible to stabilize only one orbit. When using the parametric effect, a periodic signal is applied to the system, which makes it possible to stabilize a larger number of orbits.

\section{Conclusion}

We have investigated the behavior of the square lattices of $N$ coupled quantum oscillators (Rydberg atoms) using the calculation of Lyapunov exponents spectra. We have revealed chaos in the system of $N>4$ and hyperchaos characterized by the number of positive Lyapunov exponents $M>2$ in the systems of $N>9$. We have shown that the number of positive Lyapunov exponents almost linearly depends on the number of atoms in the system, at a rate suggesting that adding three atoms leads to the appearance of an additional positive Lyapunov exponent. We have suggested using an external parametric effect and continuous feedback to control the complicated dynamics in the system. It was shown that using continuous feedback allows to reduce the number of positive Lyapunov exponents from 3 to only 2 while introducing external parametric influence into the system allows reducing their number down to 0 and completely suppresses hyperchaos. But we can suppose that using more complicated continuous feedback based on 2 or 3 circuits from different atoms can suppress hyperchaos more efficiently.

\section{Acknowledgements}

Andrey Andreev thanks Alexander Hramov and Alexander Balanov for useful discussions.

\section{References}

Andreev, A., Balanov, A., Fromhold, T., Greenaway, M., Hramov, A., Li, W., Makarov, V., and Zagoskin, A. (2019). Chaos and hyperchaos in driven interacting quantum systems. arXiv preprint arXiv:1907.03602.

Andreev, A., Moskalenko, O., Koronovskii, A., and Hramov, A. (2015). Chaos and its suppression in a system of two coupled rydberg atoms. Bulletin of the Russian Academy of Sciences: Physics, 79 (12), pp. 14321434.

Barredo, D., Labuhn, H., Ravets, S., Lahaye, T., Browaeys, A., and Adams, C. S. (2015). Coherent Excitation Transfer in a Spin Chain of Three Rydberg Atoms. Phys. Rev. Lett., 114 (11), pp. 113002.

Eastman, J. K., Hope, J. J., and Carvalho, A. R. (2017). Tuning quantum measurements to control chaos. Scientific reports, 7, pp. 44684.

Gaëtan, A., Miroshnychenko, Y., Wilk, T., Chotia, A., Viteau, M., Comparat, D., Pillet, P., Browaeys, A., and Grangier, P. (2009). Observation of collective excitation of two individual atoms in the rydberg blockade regime. Nature Physics, 5 (2), pp. 115.

Greenland, P., Lynch, S. A., Van der Meer, A., Murdin, B., Pidgeon, C., Redlich, B., Vinh, N., and Aeppli, G. (2010). Coherent control of rydberg states in silicon. Nature, $\mathbf{4 6 5}$ (7301), pp. 1057.

Heidemann, R., Raitzsch, U., Bendkowsky, V., Butscher, B., Löw, R., Santos, L., and Pfau, T. (2007). Evidence for coherent collective rydberg excitation in the strong blockade regime. Physical Review Letters, 99(16), pp. 163601.

Hramov, A. E. and Koronovskii, A. A. (2005). Generalized synchronization: a modified system approach. Phys. Rev. E, 71 (6), pp. 067201.

Hramov, A. E., Koronovskii, A. A., and Rempen, I. S. (2006). Controlling chaos in spatially extended beamplasma system by the continuous delayed feedback. Chaos, 16 (1), pp. 013123.

Ivanchenko, M., Laptyeva, T., and Flach, S. (2014). Quantum chaotic subdiffusion in random potentials. Physical Review B, 89 (6), pp. 060301. 
Jaksch, D., Cirac, J., Zoller, P., Rolston, S., Côté, R., and Lukin, M. (2000). Fast quantum gates for neutral atoms. Physical Review Letters, 85 (10), pp. 2208.

Kuznetsov, S. (2001). Dynamical chaos. Moscow: Fizmatlit.

Labuhn, H., Barredo, D., Ravets, S., de Léséleuc, S., Macrì, T., Lahaye, T., and Browaeys, A. (2016). Tunable two-dimensional arrays of single Rydberg atoms for realizing quantum Ising models. Nature, 534 (7609), pp. 667-670.

Lee, T., Häffner, H., and Cross, M. C. (2011). Antiferromagnetic phase transition in a nonequilibrium lattice of rydberg atoms. Phys. Rev. A, 84, pp. 031402.

Maximenko, V. A., Hramov, A. E., Koronovskii, A. A., Makarov, V. V., Postnov, D. E., and Balanov, A. G. (2017). Lyapunov analysis of the spatially discretecontinuous system dynamics. Chaos, Solitons \& Fractals, 104, pp. 228-237.

Mirus, K. A. and Sprott, J. (1999). Controlling chaos in low- and high-dimensional systems with periodic parametric perturbations. Physical Review E, 59(5), pp. 5313.
Ostrovskaya, E. A. and Nori, F. (2016). Giant rydberg excitons: Probing quantum chaos. Nature materials, 15 (7), pp. 702.

Pyragas, K. (1992). Continuous control of chaos, by selfcontrolling feedback. Phys. Lett. A, 170, pp. 421-428.

Pyragas, K. (1995). Control of chaos via extended delay feedback. Physics Letters A, 206 (5-6), pp. 323-330.

Pyragas, K. (1997). Conditional Lyapunov exponents from time series. Phys. Rev. E, 56 (5), pp. 5183-5188.

Saffman, M., Walker, T. G., and Mølmer, K. (2010). Quantum information with rydberg atoms. Reviews of Modern Physics, 82 (3), pp. 2313.

Schwarzschild, B. (2009). Survey of andromeda's environs reveals relics of galactic cannibalism. Physics Today, 62 (11), pp. 15.

Weimer, H., Löw, R., Pfau, T., and Büchler, H. P. (2008). Quantum critical behavior in strongly interacting rydberg gases. Physical Review Letters, 101 (25), pp. 250601.

Zagoskin, A. M. (2011). Quantum engineering: theory and design of quantum coherent structures. Cambridge University Press. 